\title{
A study of energy transfer during water entry of solids using incompressible SPH simulations
}

\author{
PRAPANCH NAIR* and GAURAV TOMAR \\ Department of Mechanical Engineering, Indian Institute of Science, Bangalore 560012, India \\ e-mail: prapanch.nair@gmail.com
}

MS received 2 May 2016; revised 1 September 2016; accepted 4 October 2016

\begin{abstract}
Cavity formation during water entry of a solid corresponds to the deceleration experienced by the solid. Several experimental studies in the past have facilitated qualitative understanding of the relation between flow and impact properties and the type of cavity formed. The types of cavities formed are classified primarily based on the nature of the seal, such as (a) surface seal, (b) deep seal, (c) shallow seal and (d) quasi-static seal. The flow mechanism behind these features and their effects on the speed of the impacting solid require further quantitative understanding. A study of such phenomenon is difficult using the existing CFD techniques owing to the fact that the high density ratios between the two phases, namely water and air, bring in issues with respect to the convergence of the linear system used to solve for the pressure field for a divergence-free velocity field. Based on a free surface modeling method, we present Incompressible Smoothed Particle Hydrodynamics (ISPH) simulations of water entry of two-dimensional solids of different shapes, densities and initial angular momenta. From the velocity field of the fluid and shape of the cavity, we relate the transfer of kinetic energy from the solid to the fluid through different phases of the cavity formation. Finally, we present a three-dimensional simulation of water entry to assert the utility of the method for analysis of real life water entry scenarios.
\end{abstract}

Keywords. Incompressible smoothed particle hydrodynamics; free surface flows; water entry; fluid-structure interaction.

\section{Introduction}

Water entry of solids has been studied for over a century. The focus of research in this field has been primarily on two aspects [1]:

1. prediction of impact loads on the body (initial stage of entry) and

2. evolution of cavity behind the falling body

The dynamics of water impact and cavity dynamics was first studied by Worthington and Cole [2]. They presented qualitative features of the splash. The problem of water entry gained immense interest during the World Wars for military projectile designs, and resulted in a number of studies [3-7] focusing on high Froude number $\left(F r=V(g D)^{-1 / 2}\right)$ regime, where the gravity effects are not important. These studies were essentially interested in the impact forces on the solid body.

For scientific and practical interests, and for civil applications, the studies later expanded to consider lower Froude numbers, where the effect of gravity is comparable to inertial effects. The problem finds significance in the study

*For correspondence of ship slamming, air to sea ballistics for anti-torpedo defence and in the study of biological creatures utilizing hydrodynamics to walk on water, for example the locomotion of basilisk lizard [8]. Also, water entry is of considerable importance in various off-shore and marine industries such as oil refineries and offshore wind farms as various components of the structure in the splash zone of the incident waves would be frequently entering (and exiting) the water surface. It was observed that the air cavity, forming behind the projectile, influences its dynamics and trajectory at such low speed water entry $[1,9,10]$. Extensive studies on water entry of spherical rigid bodies have been performed by Truscott et al [11]. They studied the splash and the Magnus effect on spinning spheres entering water (see $[12,13]$ ), the effect of different types of surface coating (hydrophilic and hydrophobic) on the dynamics of the cavity formation in [14] and the dynamics of skipping stones in [15]. Various numerical and theoretical studies have also been performed. Birkhoff et al [16] used 2D analytical models using an arbitrary constant to account for 3D scenarios. All the analytical studies use empirical constants to determine the kinetic energy of the fluid in the expansion phase, when the radius of the cavity continues to increase at each vertical location. Numerical studies of water entry face the difficulty in modelling two- 
way coupled simulation involving the rigid bodies together with the interaction of the interface between air and water. Grid-based simulations methods such as volume of fluids $(\mathrm{VoF})$ and front tracking methods have had some success in simulating such problems [17]. However, complex flow phenomena such as breaking waves and fragmented drops of fluid due to the impacting solid are a challenge to handle using the grid-based numerical methods.

Smoothed Particle Hydrodynamics (SPH) is a meshless Lagriangian-particle-based flow simulation method introduced in [18, 19]. A vast account of its applications and challenges can be found in [20]. Though traditional SPH algorithms use a stiff equation of state to model incompressibility as weak compressibility, a purely incompressible variant, which solves for pressure that ensures divergence-free velocity field, was proposed in [21]. In Ref. [22] an issue with using divergence-free velocity as the condition for incompressibility was pointed out and a deformation-gradient-based algorithm for closing the governing equations of the system was proposed. SPH simulations have been used extensively in simulating free surface phenomena; however purely incompressible SPH methods faced difficulties in imposing Dirichlet boundary condition $(\mathrm{BC})$ at the free surface. This was overcome in [23] by introducing a semi-analytical method to apply Dirichet $\mathrm{BC}$ at the free surface. With this free surface modelling, the application of Dirichlet BC at the free surface is straightforward, and enables one to solve problems like water entry of solids with greater accuracy.

In this paper, we first explain modelling of rigid body dynamics and its interaction with the fluid using SPH and subsequently discuss the validation of the model against experiments by Greenhow and Lin [24]. Motivated by the recent experiments by Truscott et al [11], Truscott and Techet [25] and Aristoff et al [26], we conduct numerical experiments of water entry of solids of different shapes with and without imparted spin. We perform a range of simulations in two dimensions with varying parameters such as aspect ratio (shape) of the impacting solid and the imparted spin. In these cases, we maintain the same kinetic energy for the impacting solid. The parametric study is limited to two dimensions due to computational constraints on performing simulation for such a wide range of parameter values. Finally, we present a 3D simulation of water entry of a sphere, where the deceleration upon impact of the sphere is compared to computations based on an analytical model of the impact.

\section{SPH formulation}

The incompressible formulations of SPH is discussed in this section. We use bold notation for vectors and tensors throughout this paper.

\subsection{SPH approximation}

In the SPH method, fluid domain is discretized into constant-mass particles, which are treated as interpolation points as well. These interpolation points have an associated kernel function with an appropriate support. This kernel function is a smoothed Dirac-delta function, and hence a variable $A(\mathbf{r})$ at a position $\mathbf{r}$ can be represented as a convolution product of the field $A$ and the kernel function over the domain:

$$
A(\mathbf{r})=\int A\left(\mathbf{r}^{\prime}\right) W\left(\mathbf{r}-\mathbf{r}^{\prime}, h\right) d \mathbf{r}^{\prime},
$$

where $W(r, h)$ is the interpolating kernel, $h$ is the smoothing length parameter and $\mathbf{r}$ is the location where property $A$ is to be evaluated. Primed notation refers to the neighbourhood of the position in question. The kernel function $W$ has the following properties:

$$
\begin{gathered}
\int W\left(\mathbf{r}-\mathbf{r}^{\prime}, h\right) d \mathbf{r}^{\prime}=1, \\
\lim _{h \rightarrow 0} W\left(\mathbf{r}-\mathbf{r}^{\prime}, h\right)=\delta\left(\mathbf{r}-\mathbf{r}^{\prime}\right),
\end{gathered}
$$

where $\delta$ is the Dirac delta function. The above interpolant in SPH is approximated by a summation over the surrounding particles:

$$
A(\mathbf{r}) \approx \sum_{b} m_{b} \frac{A_{b}}{\rho_{b}} W\left(\mathbf{r}-\mathbf{r}_{b}, h\right),
$$

where the index $b$ denotes a particle in the neighbourhood of the particle at position $\mathbf{r}$, which has mass $m_{b}$, position $\mathbf{r}_{b}$, density $\rho_{b}$, velocity $\mathbf{v}_{b}$ and the variable value $A_{b}$. Derivatives of functions can be approximated in several conservative and non-conservative ways [20]. A symmetric and conservative gradient approximation often used to approximate pressure gradients is given by

$$
\nabla A_{a}=\rho_{a} \sum_{b} m_{b}\left(\frac{A_{b}}{\rho_{b}^{2}}+\frac{A_{a}}{\rho_{a}^{2}}\right) \nabla_{a} W_{a b}
$$

The order of the kernel affects the accuracy of the solution and the resolution of the properties at different length scales. Furthermore, particle approximation transforms the integration over the kernel to summation and hence invokes integration errors [27-29]. Using the aforementioned formulation, smoothed fields can be obtained and the corresponding partial differential equations can be solved for the field values at the interpolation points. In what follows, we discuss the governing equations for incompressible free surface flows and the ISPH algorithm for solving the equations. 


\subsection{Governing equations}

Momentum conservation equations for a Newtonian fluid are solved using the SPH method in the Lagrangian framework. Navier-Stokes equations governing the momentum conservation are given as follows:

$$
\frac{d \mathbf{u}}{d t}=\frac{1}{\rho}\left(-\nabla p+\nabla \cdot(2 \mu \mathbf{D})+\mathbf{f}^{B}\right),
$$

where $\mathbf{u}$ is the velocity, $p$ is the pressure, $\rho$ and $\mu$ are the density and coefficient of viscosity of the fluid, respectively, $\mathbf{D}=\left(\nabla \mathbf{u}+\nabla \mathbf{u}^{T}\right) / 2$ is the deformation rate tensor, $\mathbf{f}^{B}$ is the body force per unit mass on the fluid element and $t$ denotes time. The Navier-Stokes equation has been written in the Lagrangian formulation and $d / d t$ denotes the material derivative. Mass conservation equation is given as follows:

$$
\frac{1}{\rho} \frac{d \rho}{d t}+\nabla \cdot \mathbf{u}=0
$$

\subsection{ISPH formulation}

For incompressible fluids, the material derivative of density is zero and therefore, condition for incompressibility is given by $\nabla \cdot \mathbf{u}=0$. In the weakly compressible formulation of SPH (WCSPH) [20, 30], an equation of state relating pressure field with density is employed that allows fluctuations in the density field. For example, the following equation of state is generally used for water [31]:

$$
p=\frac{\rho_{0} c_{0}^{2}}{\gamma}\left(\left(\frac{\rho}{\rho_{0}}\right)^{\gamma}-1\right),
$$

where $\rho_{0}$ and $c_{0}$ are the reference density and speed of sound, respectively, and $\gamma=7$ for water. Small errors incurred in particle positions and therefore in density are amplified by Eq. (8) and lead to large fluctuations in the pressure. To circumvent this issue for incompressible flows, a projection algorithm in which pressure field is obtained by solving a Poisson equation was proposed by Cummins and

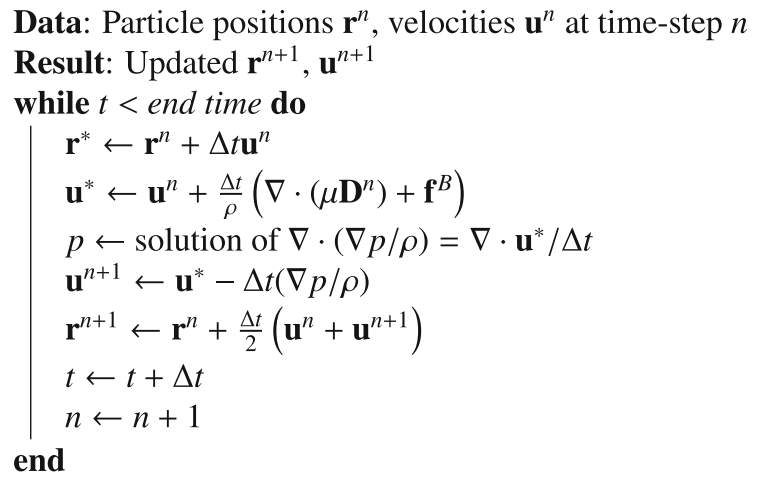

Algorithm 1 ISPH algorithm with predictor-corrector time integration for position [32].
Rudman [21]. This scheme, popularly known as Incompressible Smooth Particle Hydrodynamics (ISPH), is given by the following algorithm.

This algorithm requires the solution of the SPH approximation to the pressure Poisson equation (PPE).

\subsection{SPH approximation of the pressure Poisson equation}

A finite-difference-based second-order derivative approximation was provided by [21] and is being widely used for approximating the Laplacian terms in the PPE. The approximation is given by the relation

$$
\nabla \cdot\left(\frac{\nabla P}{\rho}\right)_{a}=\sum_{b} \frac{m_{b}}{\rho_{b}} \frac{4}{\rho_{a}+\rho_{b}}\left(P_{a}-P_{b}\right) F_{a b} .
$$

This is a non-conservative approximation for interaction of the particle pair $a$ and $b$. Considering each particle $a$ and its neighbours $b$, this results in a symmetric sparse linear system of equations, which we solve by a Krylov solver such as BiCGSTAB using the LIS linear solver library [32]. The RHS of the PPE can be approximated conservatively [21] as follows:

$$
\frac{\nabla \cdot \mathbf{u}_{a}}{\Delta t} \approx \frac{\rho_{a}}{\Delta t} \sum_{b} m_{b}\left(\frac{\mathbf{u}_{b}}{\rho_{b}^{2}}+\frac{\mathbf{u}_{a}}{\rho_{a}^{2}}\right) \cdot \nabla_{a} W_{a b}
$$

or non-conservatively as follows:

$$
\frac{\nabla \cdot \mathbf{u}_{a}}{\Delta t} \approx-\frac{1}{\rho_{a} \Delta t} \sum_{b} m_{b}\left(\mathbf{u}_{a}-\mathbf{u}_{b}\right) \cdot \nabla_{a} W_{a b} .
$$

For internal flow problems where the PPE has homogeneous Neumann BC on all boundaries, only a conservative divergence approximation satisfies the integral constraint and ensures existence of a solution [21]. However, free surface flow problems have Dirichlet and Neumann BCS for the PPE and the RHS of PPE does not have to satisfy any constraint. Moreover, the conservative divergence approximation introduces errors near the free surface due to kernel truncation. Hence, the non-conservative approximation for divergence of velocity (Eq. 11) is appropriate for free surface problems.

In the following section we discuss the imposition of BCS for PPE at the free surface.

\section{Free surface boundary conditions}

In ISPH methods, particles at the free surface are traditionally identified explicitly based on either the change in the density, colour function or by using the kernel support deficiency $[33,34]$. A certain criterion is generally chosen to distinguish the free surface particles from the bulk particles such as $\rho<0.99 \rho_{0}$ [33] or $\sum_{b} W\left(\mathbf{r}_{a}-\mathbf{r}_{b}, h\right)<0.97$ 


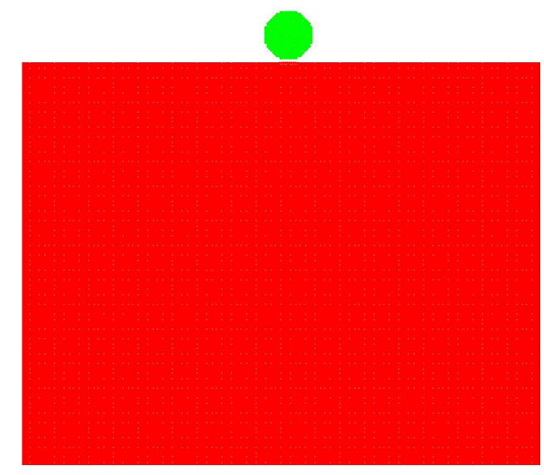

(a) $t=0.0025 \mathrm{~s}$

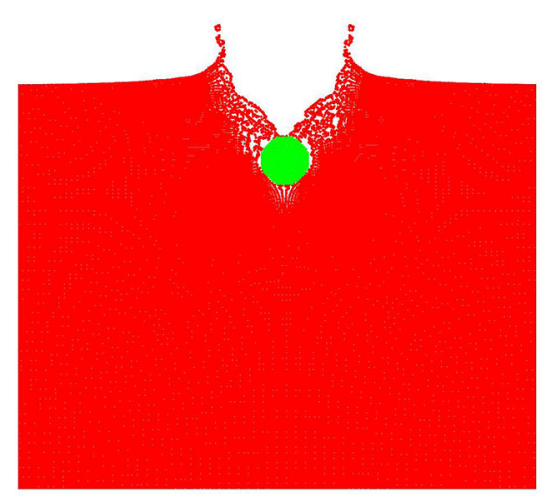

(d) $t=0.04 \mathrm{~s}$

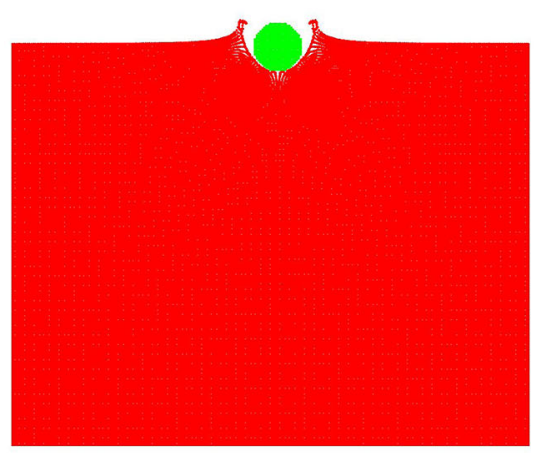

(b) $t=0.012 \mathrm{~s}$

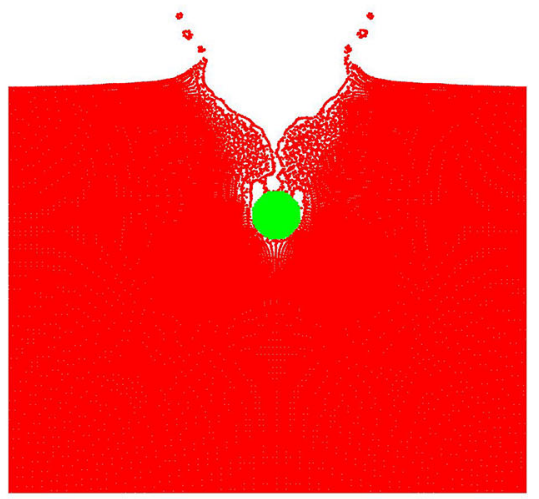

(e) $t=0.07 \mathrm{~s}$

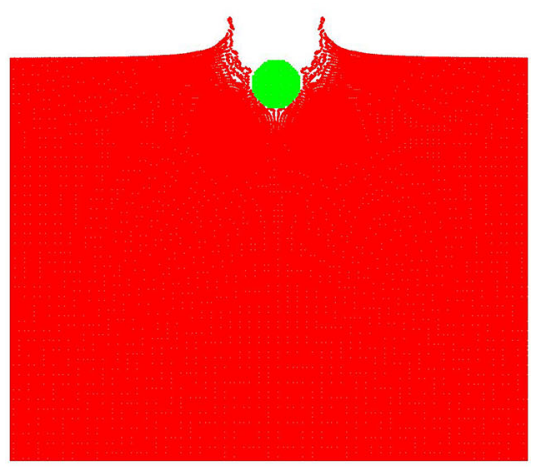

(c) $t=0.02 \mathrm{~s}$

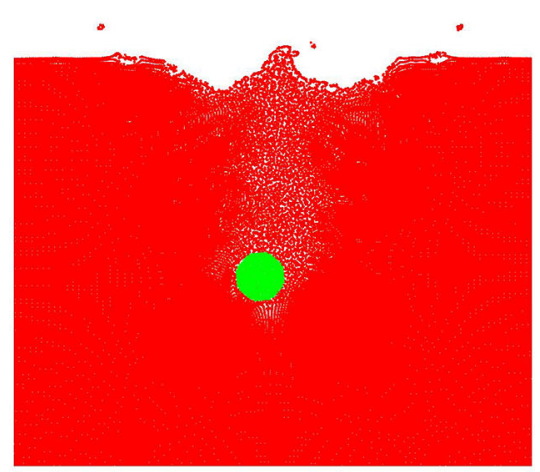

(f) $t=0.15 \mathrm{~s}$

Figure 1. Water entry of rigid bodies of aspect ratio 1.0 (circular cylinder).

[35]. Another technique is to use the ratio of number of neighbours around a particular particle to the total number of neighbours expected in the interior $[36,37]$. The divergence of the position vector $(\nabla \cdot \mathbf{r})$ of particles has also been used to identify free surfaces [38, 39]. For 2D and 3D simulations, $\nabla \cdot \mathbf{r}$ takes values 2 and 3 , respectively, for interior particles. A value of 1.5 is used to demarcate the free surface particles for 2D simulations. However, a very low threshold value of these parameters, due to slight errors in particle configurations, can lead to incorrect marking of interior points as free boundary, whereas, if a very high threshold value is used, some free surface particles would be treated as interior particles. Therefore, choosing an appropriate threshold value is critical and introduces arbitrariness in the simulation. A semi-analytical method to impose a constant pressure $\mathrm{BC}$ at free surfaces was proposed in [23]. This method also allows for pressure gradients along the free surfaces. In the present work we use this method to apply the pressure BC at free surface.

\subsection{Solid wall boundary conditions}

In this study, we use the method proposed by Koshizuka et al [37] in the context of solid wall BCS for the Moving Particle
Semi-Implicit method. The method essentially involves using static particles with same properties as those of fluid particles to represent solid wall boundaries. The pressure equation is also solved on these static wall particles. As the divergence is zero within the solid walls (due to zero velocity), the method tends to simulate a diffused interface between the fluid and the solid only to the order of the smoothing kernel of particles. By including a few layers of wall particles in the domain of the Poisson solver, we effectively impose homogeneous Neumann BCS at the walls.

\subsection{Rigid body formulation}

Motion of rigid bodies can be modelled by computing the net fluid force and torque acting on them. We model the rigid body with SPH particles as well. For a rigid body with the centre of mass at $\mathbf{R}$ and translational velocity $\mathbf{V}$, the equation of motion for the centre of mass is given by

$$
M \frac{d \mathbf{V}}{d t}=\sum_{k} m_{k} \mathbf{a}_{k},
$$

where $M$ and $m_{k}$ are the mass of the rigid body and mass of the SPH particles representing the rigid body, respectively. 


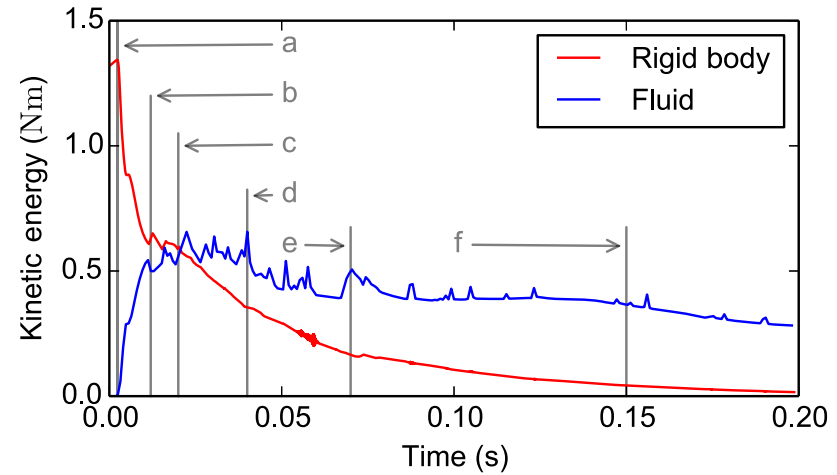

Figure 2. Kinetic energy of rigid body on water entry and the imparted kinetic energy to the fluid. Labels correspond to those of figure 1 .

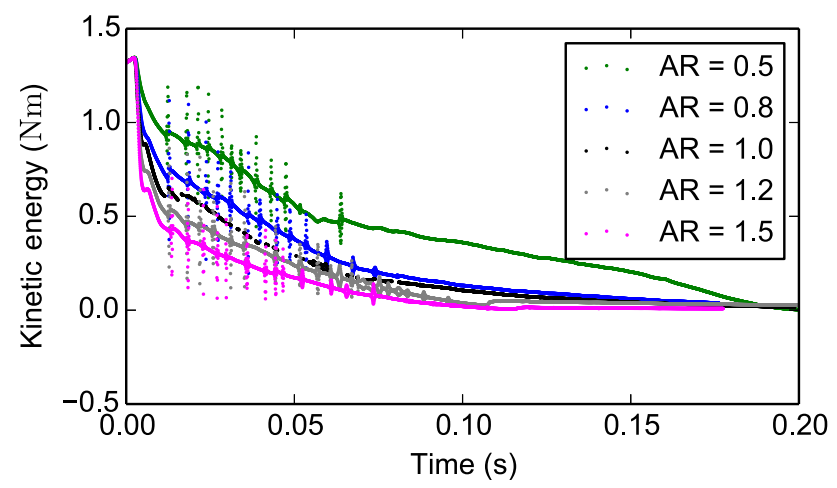

Figure 3. Kinetic energy of impacting elliptic cylinders with different aspect ratios.

The acceleration of the particle $k$ is $\mathbf{a}_{k}$. The position and velocity of the SPH particles constituting the solid are updated using the rigid body acceleration $d \mathbf{V} / d t$ and velocity $\mathbf{V}$, respectively, instead of using the acceleration $\mathbf{a}_{k}$ that is based on the local force on the particles.

Conservation of angular momentum of a rigid body is given by

$$
I \frac{d \Omega}{d t}=\tau,
$$

where $I$ is the moment of inertia, $\Omega$ is the angular velocity and $\tau$ is the torque. Using particle approximation, Eq. (13) can be written as

$$
I \frac{d \Omega}{d t}=\sum_{k} m_{k}\left(\mathbf{r}_{k}-\mathbf{R}\right) \times \mathbf{a}_{k},
$$

where $\mathbf{r}_{k}$ is the position of the particle $k$. Since the rigid body moves as a whole, its particles change their positions according to

$$
\frac{d \mathbf{r}_{k}}{d t}=\mathbf{V}+\Omega \times\left(\mathbf{r}_{k}-\mathbf{R}\right)
$$

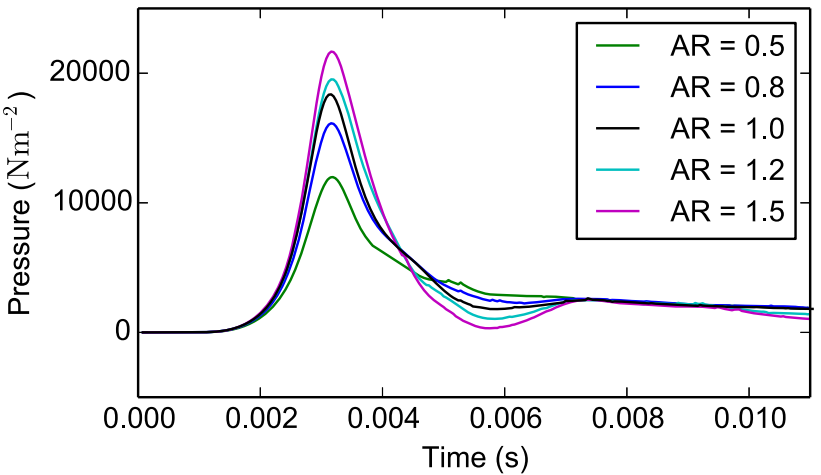

Figure 4. Impact pressure at cylinder bottom for different aspect ratios.

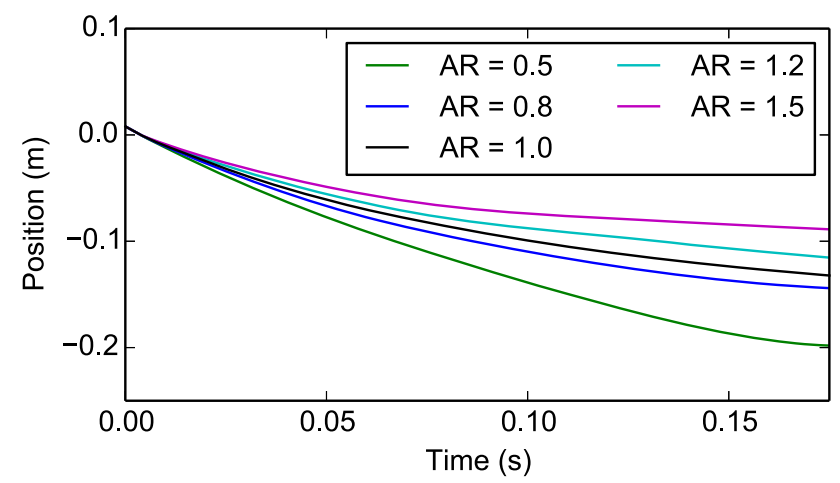

Figure 5. Position of cylinder during water entry for different aspect ratios.

Monaghan [20] showed that the algorithm discussed earlier for velocity computation for rigid body conserves linear and angular momentum in SPH approximations for rigid body motion.

Since the pressure correction equation is solved in a region including the rigid body, the pressure field exists within the rigid body as well, and using the divergence theorem we get

$$
\int_{S} p d S=\int_{V} \nabla p d V .
$$

Hence the total force on the rigid body can be computed from the SPH approximation of the RHS of Eq. (16). Similarly, viscous forces are computed on individual solid particles and integrated to give the total force and torque on the whole solid. The free surface correction of the Laplacian term is not performed for the particles representing rigid bodies. The acceleration $\mathbf{a}_{k}$ for the particle $k$ essentially comprises the components from the forces discussed earlier. 

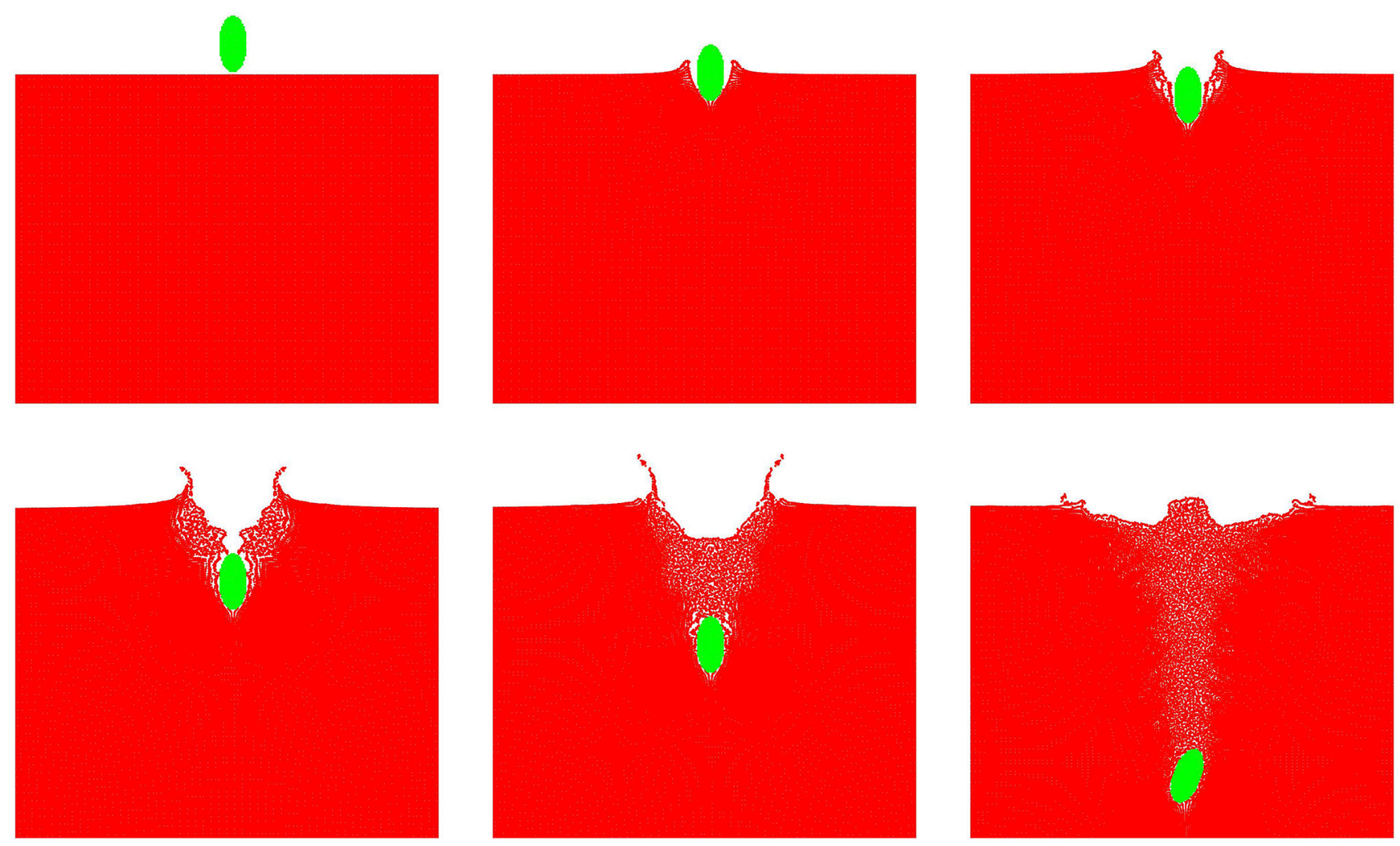

Figure 6. Water entry of rigid bodies of aspect ratios 0.5.
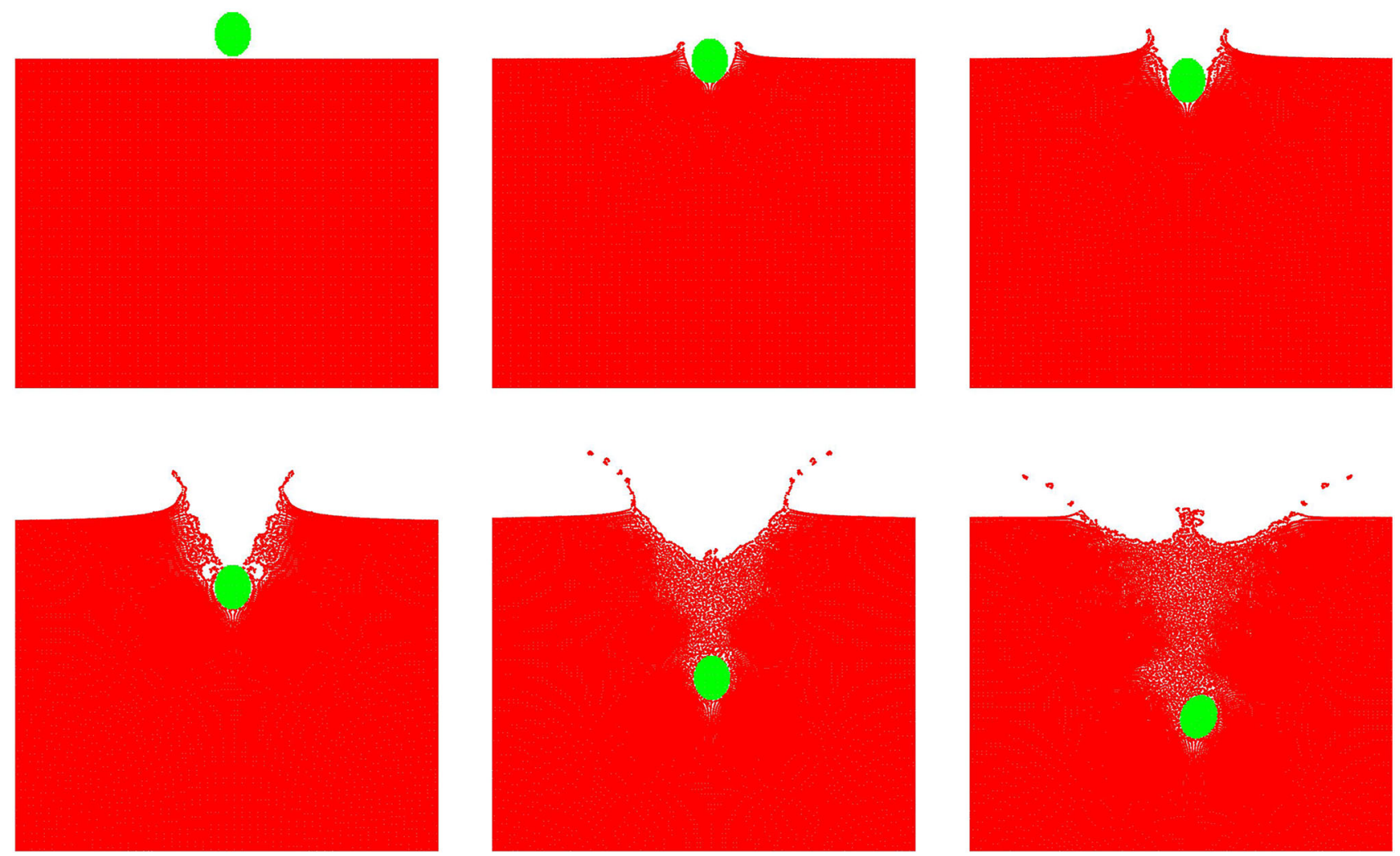

Figure 7. Water entry of rigid bodies of aspect ratio 0.8 . 

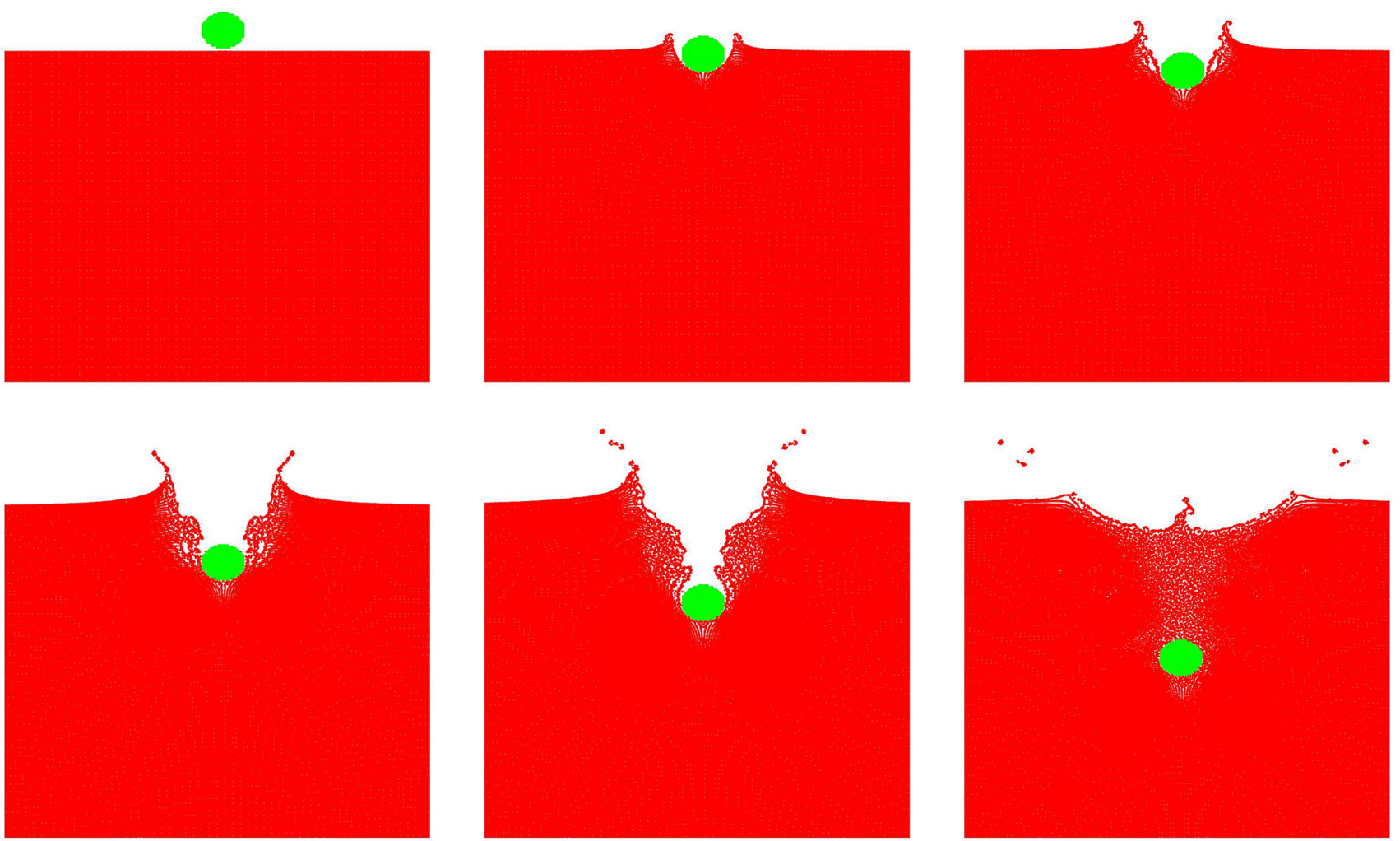

Figure 8. Water entry of rigid bodies of aspect ratio 1.2.

\section{Results and discussion}

The SPH algorithm presented earlier with the implementation of free surface BC can be used to study water entry of solids with all degrees of freedom encountered in real life scenarios. We present simulations of water entry of solids with varying shapes, angular velocities and densities and present observations on the splash and energy transfer to the liquid body. A 3D simulation is also presented and compared to the solution of an analytical model for water entry in this section.

\subsection{Water entry of cylinders of varying shapes}

In this section, we look at the simulated interface, pressure and energetics of water entry of a cylinder (2D) for different aspect ratios. We begin by considering the water entry of a circular cylinder of 1-in diameter (simulated in 2D with no restriction on degrees of freedom) into a still fluid of density $1000 \mathrm{~kg} / \mathrm{m}^{3}$ (see figure 1). The Froude number for this problem is $\sim 4.6$. As the solid impacts the free surface, two jets emerge from both sides and they begin to expand. A cavity is formed behind the cylinder (see figure 1c and d). The jets fold inwards towards the cylinder after the cavity starts collapsing. In figure 1e we see the two walls of the cavity come together to form a seal. This is described as the deep seal, characteristic of the low
Froude number regime. The present simulations do not account for the presence of air in the cavity and hence the seal is observed very close to the cylinder, which may be different from the actual physical case. However, we continue the simulation to observe the free surface dynamics. After the cavity collapses, we see a small jet (see figure 1f) at the middle of the surface. In 3D, this jet would have had a greater height due to the focusing effect. The kinetic energy transfer from the solid to fluid is depicted in figure 2 and the various stages are annotated in the graph.

The kinetic energy transfer from the solid to fluid is shown in figure 2. The points labelled correspond to different stages of the cavity development and collapse shown in figure 1. After impacting the water surface, the projectile transfers its kinetic energy to the fluid until the point (b), when the initial jets of the fluid emerge from the neck of the impact. The region from (c) to (d) marks the separation of the fluid from the solid, when the kinetic energy of the fluid varies little. The region (d) to (e) represents the expansion and collapse of the cavity with the minima in this region corresponding to an instance when the cavity is the widest. A deep seal of the cavity is observed at (e) and is marked by a local surge in the kinetic energy of the fluid. We expect the flow to deviate from reality at this point due to the absence of air in the simulations. Subsequent to the collapse of the cavity, a progressive drop in kinetic energy is observed beyond (f), 

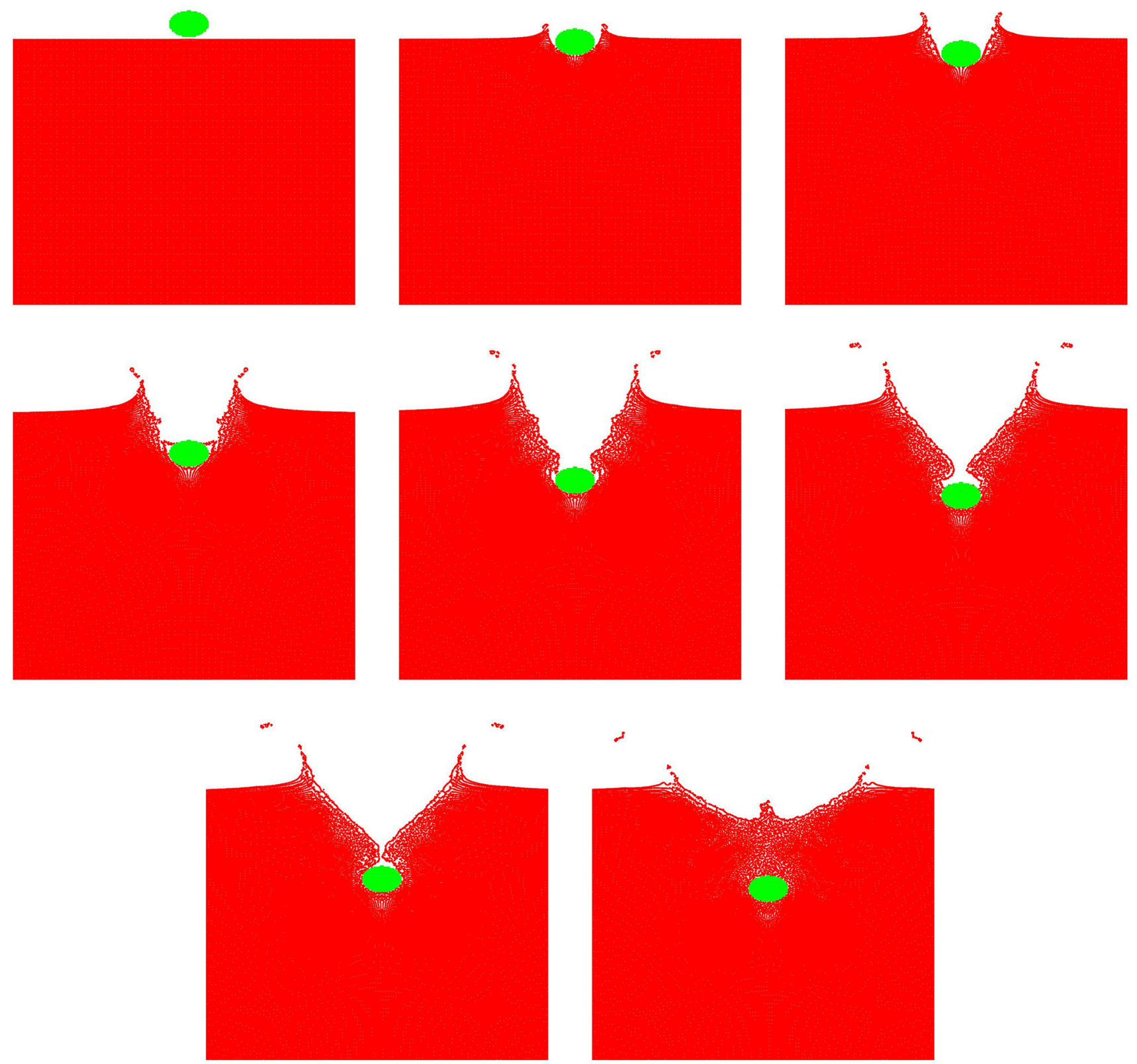

Figure 9. Water entry of rigid bodies of aspect ratio 1.5.

which suggests dissipation of energy due to viscous effects.

We now present the simulations of water entry of elliptic cylinders for different aspect ratios to look at how the splash zone, cavity and the impact pressure on the solid change with the shape of the impacting solid. We choose elliptic cylinders of five different aspect ratios, 0.5, 0.8, 1.2, 1.5 and the circular cylinder presented earlier, with the same mass and density. Here, aspect ratio is the ratio of horizontal measure (width) to the vertical measure (height) of the solid. Figure 3 shows the kinetic energy variation of the cylinders. The graphs show oscillations of the computed value. The sharp oscillations observed are essentially numerical and correspond to flow separation at the point where free surface meets the solid surface and the values oscillate about a local mean. These spurious oscillations can be controlled using higher order time stepping schemes. The energy drop happens at a slower rate for the solid of AR 0.5, at the initial part of the entry. This can be explained due to its more streamlined shape. The pressure force on the solid is measured at the bottom of the bodies as shown in figure 4. The variation in pressure peaks experienced by different shapes essentially corresponds to the streamlined shape of the body. Subsequently the pressure decreases continuously as the solid body enters further into the fluid. An inflection point is observed in pressure curve, which 


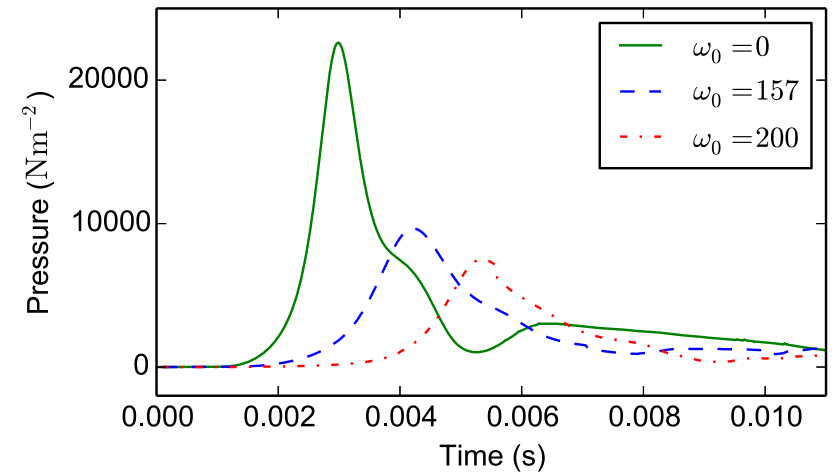

Figure 10. Impact pressure at water entry of rigid body with different angular velocities.

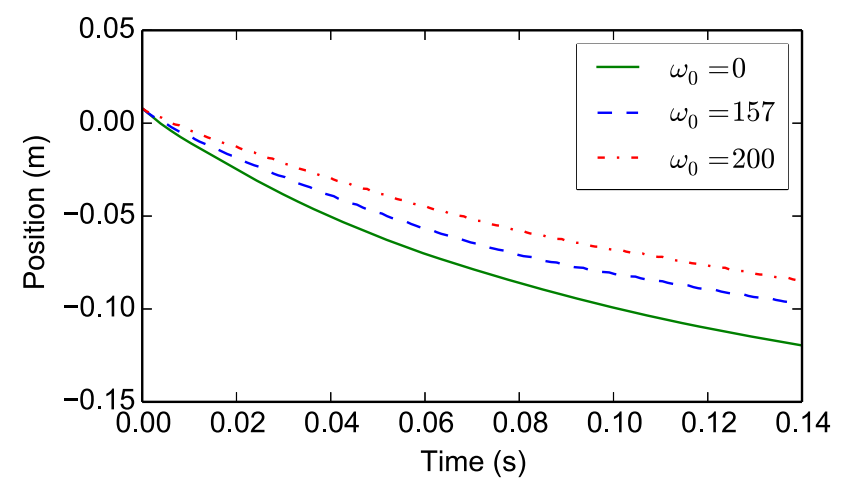

Figure 11. Position of rigid bodies with different angular velocities during water entry.

marks a sudden decrease in the slope of the curve and is more remarkable for low aspect ratios. Further, decrease in pressure is observed with a minimum occurring for aspect ratios greater than unity. Beyond this minimum, the pressure again increases slightly. Interestingly, all the pressure curves merge at around $0.007 \mathrm{~s}$, essentially because the pressure force balances the reaction force of the fluid to solids of equal mass impacting on its surface. Subsequently, the pressures deviate based on the coefficient of form drag for the different shapes.

Figure 5 shows the position of a cylinder during water entry and figures 6-9 show the different stages of water entry of the cylinders for different aspect ratios at different times. A prolate-shaped particle shows small amplitude rotations during its motion in the liquid pool (see figure 6). Figure 7 shows a small change in the nature of cavity closing behind the particle with an increase in the circularity of the particles (from 0.5 in figure 6 to 0.8 in figure 7). The cavity becomes wider with an increase in the cylinder-aspect ratio (see figures 8 and 9). For an aspect ratio of 1.2, the cavity shows formation of stepped profile whereas for aspect ratio of 1.5 , a more well-formed triangular shape is observed, similar to the one for circular cylinders.

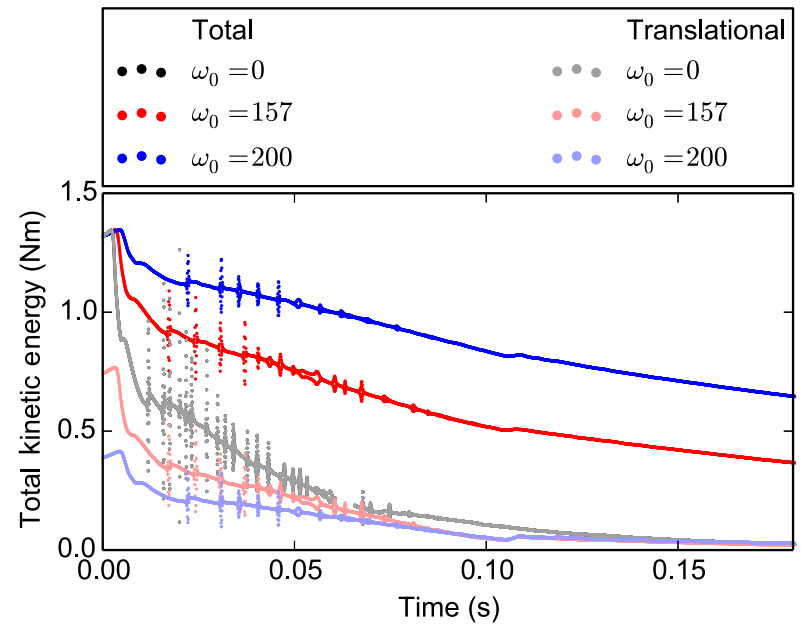

(a)

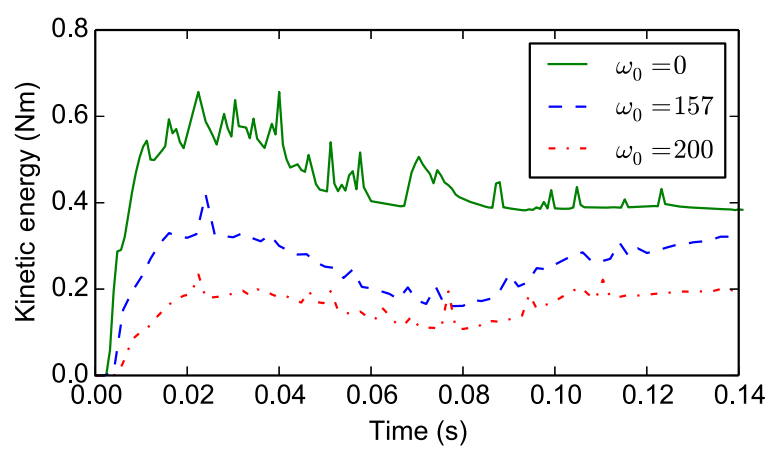

(b)

Figure 12. Energy transfer between solid and fluid with varying angular velocities. (a) Kinetic energy (total and translational) of rigid body. (b) Kinetic energy imparted to the fluid.

\subsection{Varying spin}

In this section we consider the water entry of cylinders with different angular velocities. For the same geometry and fluid properties considered earlier, we spin the cylinders with an initial angular velocity of 157 and $200 \mathrm{Rad} / \mathrm{s}$, respectively, while reducing the velocity correspondingly such that the total kinetic energy of the cylinders before impact remains the same. We note that the cylinders consist of SPH particles, and these particles are set to have a high viscosity coefficient to make the interface a close approximation to a no-slip wall.

As seen in figure 10 the impact pressure of the cylinders with finite angular velocity is lesser due to the reduced translational kinetic energy. Also the inflection point after the impact disappears with imparted spin. Figure 11 shows the variation in penetration of the solids with different initially imparted spins. Clearly, for a higher initial spin case, translational energy is less and therefore we observe a slower rate of penetration. Figure 12 shows both the translational and total kinetic energy of the cylinders. The 

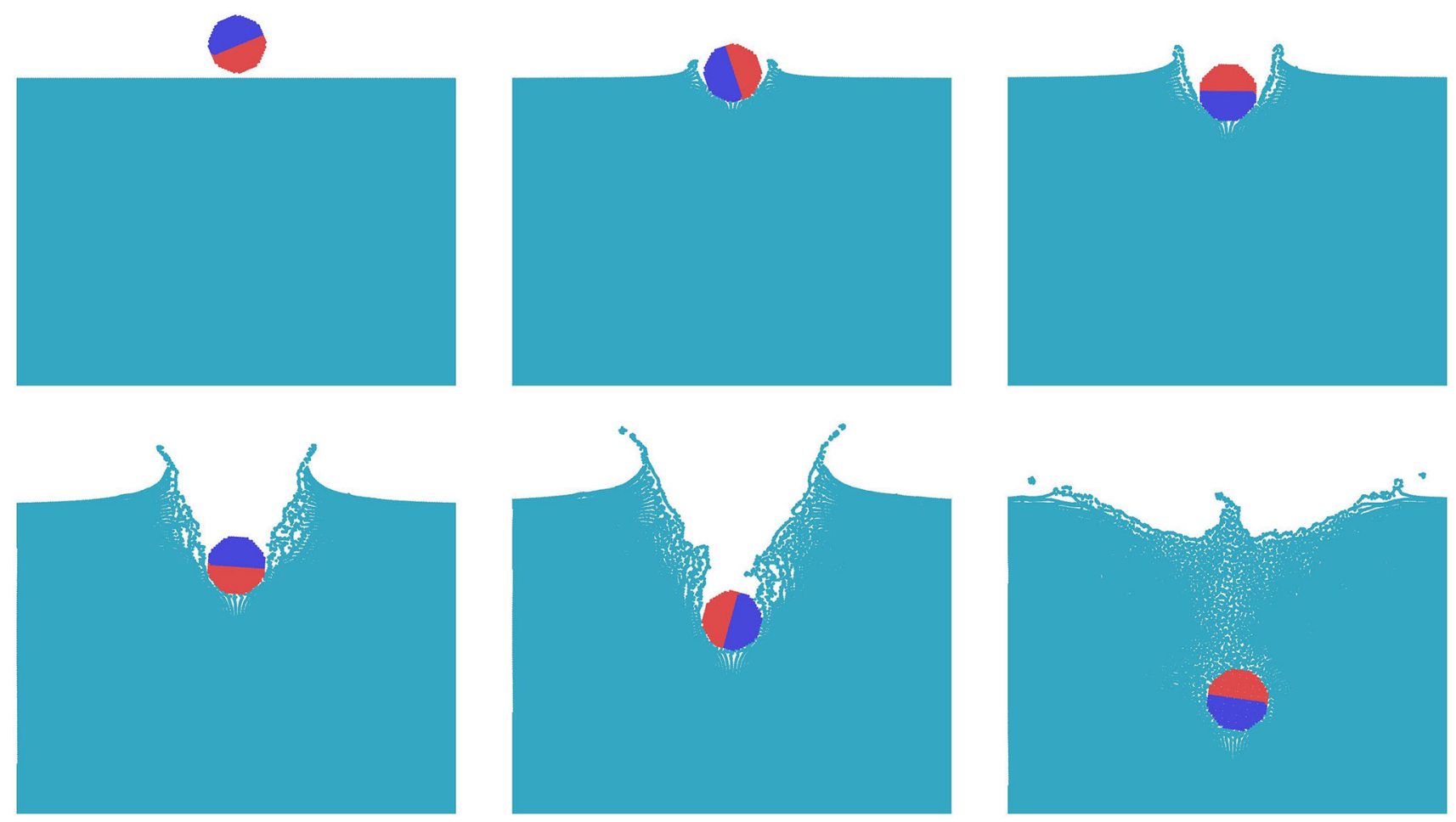

Figure 13. Water entry of cylinder spinning at $\omega_{0}=157$.
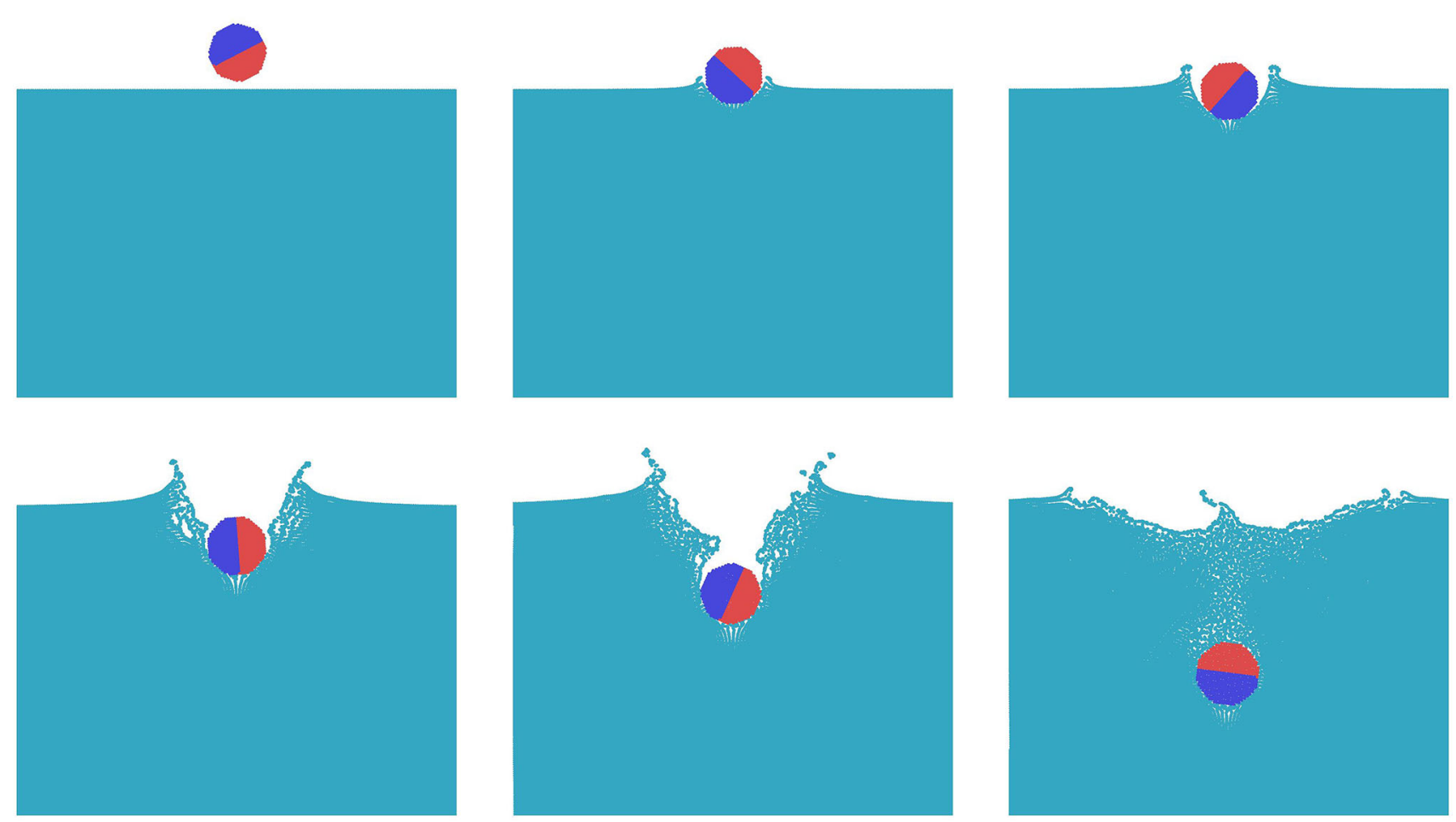

Figure 14. Water entry of cylinder spinning at $\omega_{0}=200$. 


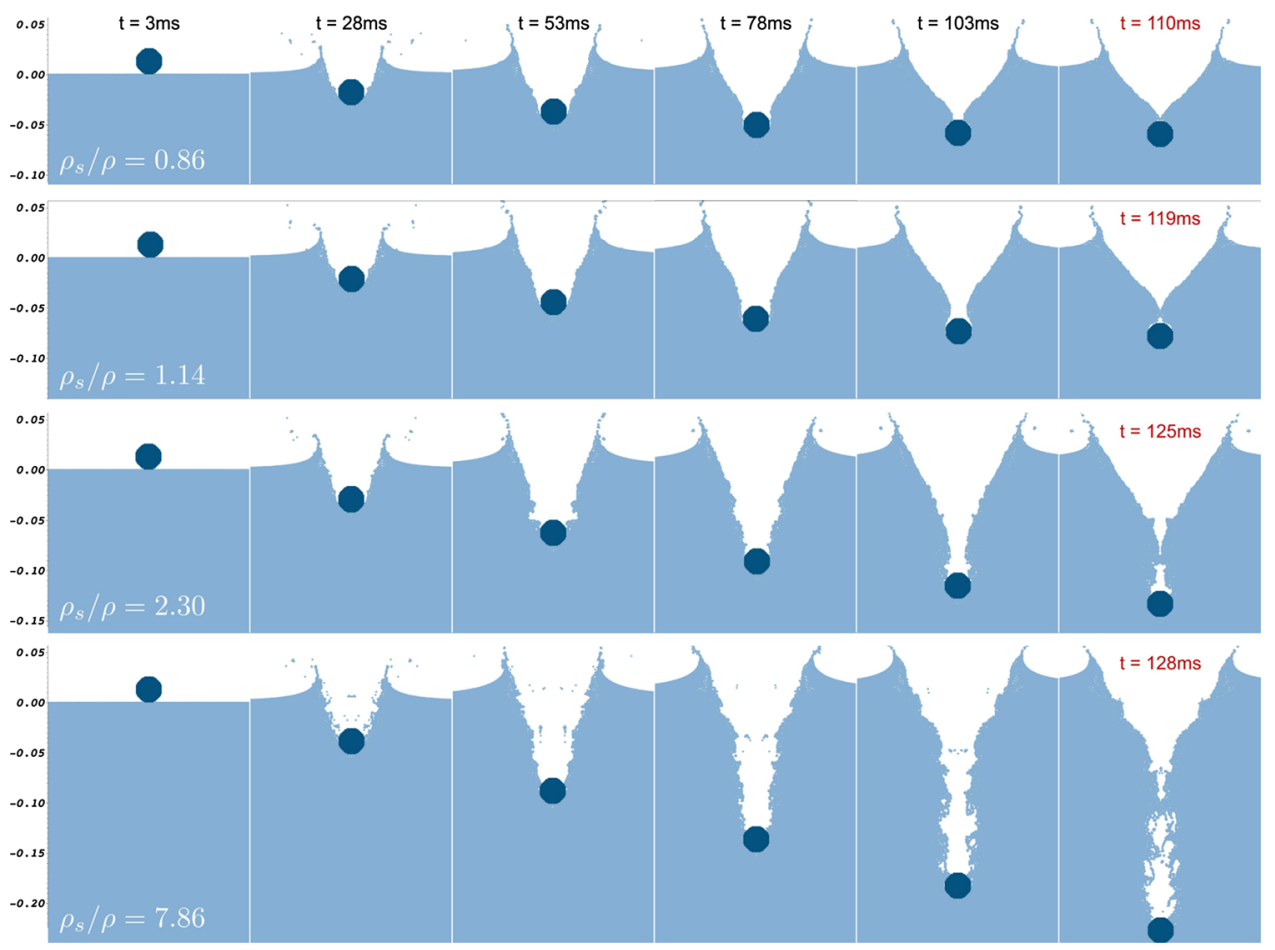

Figure 15. Water entry of cylinders of different densites.

total kinetic energy of the cylinders is transferred the least to the fluid when the spin velocity is high. The kinetic energy of the fluid is shown in figure 12b. For the case with zero spin, the kinetic energy, after reaching its peak, decreases down to a local minimum as explained earlier. However, for the cases with spin, the local minimum is more pronounced and delayed, suggesting a delayed instance of maximum cavity volume. The kinetic energy rises considerably after this minimum and approaches the kinetic energy of fluid in the case without spin.

\subsection{Varying solid density}

In the study of decelerating spheres by Aristoff et al [26], spheres of different density were considered. We conduct simulations of different density cylinders with the same velocity of entry. Figures 13-15 show the different stages of water entry in our simulations. In general, the increase in density causes the pinch-off to happen later in time and farther from the cylinder. For cases that are close to neutral buoyancy, the pinch-off is predicted to happen rather close to the solid cylinder than shown in experiments (figure 3 of [26]). Effect of presence of air could be the reason for this. Also the experiments in [26] are conducted for spheres and there is a focusing effect due to three dimensionality of the shape. Another noticeable aspect of these simulations is that as the density increases, the undulations on the surface of the cavity are more pronounced and lead to filament-like breakup of the free surface. This could be because of the two dimensionality of the simulation, and requires further attention.

\subsection{Three-dimensional simulation}

We have conducted a 3D simulation of water entry. In this simulation we use about one million particles and it is therefore computationally intensive. We use a shared memory parallelization to parallelize this case. Owing to the computational limitation the simulation is limited to low Froude number $(\sim 3.2)$ with impact velocity of $1.6 \mathrm{~m} /$ s. Figure 16 is a ray-traced render of the simulation of water entry of a 1-in sphere entering the surface of water at $\mathrm{Fr} \approx$ 3.2 and with an impact velocity of $1.6 \mathrm{~m} / \mathrm{s}$. The sphere is set to be neutrally buoyant. In agreement with the experiments of [26], very little splash is observed in the simulation at such a low Froude number. As the sphere enters the water surface, the cavity shows formation of waves on the free surface. The cavity then pinches off very close to the sphere, characterizing a deep seal. Figure 17 shows the impact pressure at the bottom-most point of the sphere. After a peak pressure is reached, the pressure drops till about $0.006 \mathrm{~s}$ and reaches a plateau for a short duration, after which the pressure declines steadily. Figure 18 shows the decrease in downward velocity of the sphere. After the initial steep decrease in velocity, the reduction in velocity slows down and reaches a plateau at $0.1 \mathrm{~s}$. Buoyancy governs the later part of the velocity change. The deceleration of the sphere can be computed from the momentum 


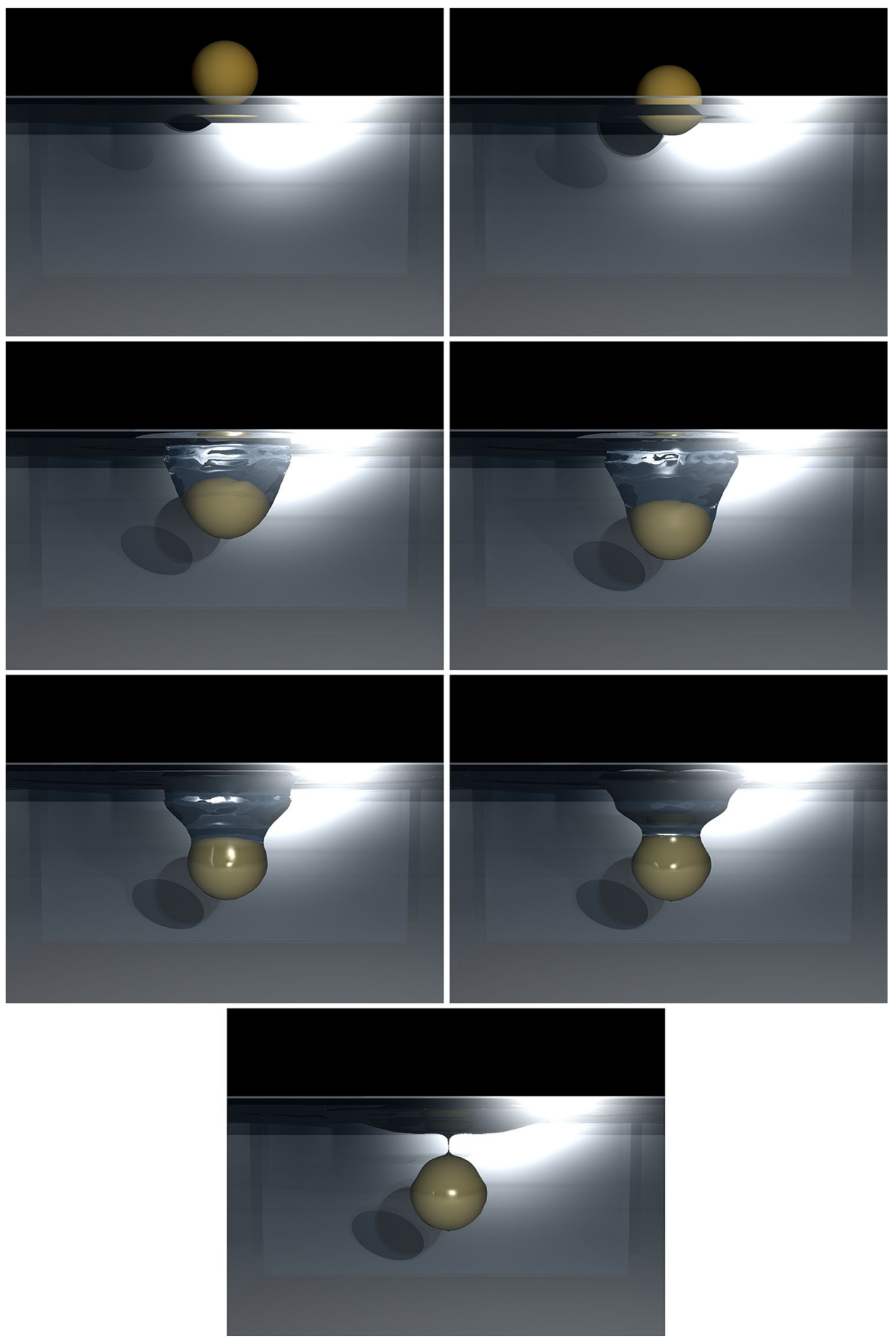

Figure 16. Water entry of a sphere at $F r=3.2$.

equations based on the theory proposed by von Karman during his investigation into the stress analysis of sea plane floats [40, 41], and this can be used as a first approximation to the computed values of acceleration from the SPH simulation. The vertical velocity of the sphere drops exponentially (see figure 18) and reaches a zero very soon and is expected to have a short duration of upward velocity.
Figure 19 shows the acceleration of the sphere as it impacts the water surface. A theoretical estimate of the acceleration can be derived as follows: the momentum balance at a time $t$ during the water entry of the sphere can be written as

$$
m V_{0}=m V+f(a) V
$$




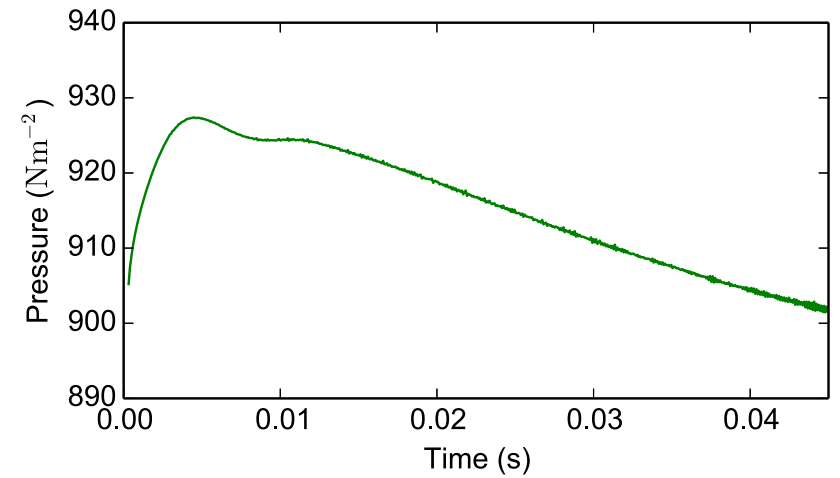

Figure 17. Impact pressure of sphere on water entry.

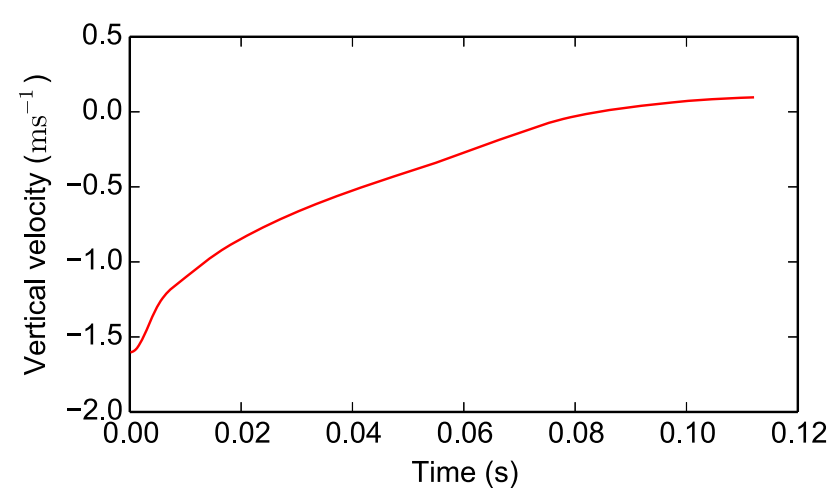

Figure 18. Vertical velocity of sphere on water entry.

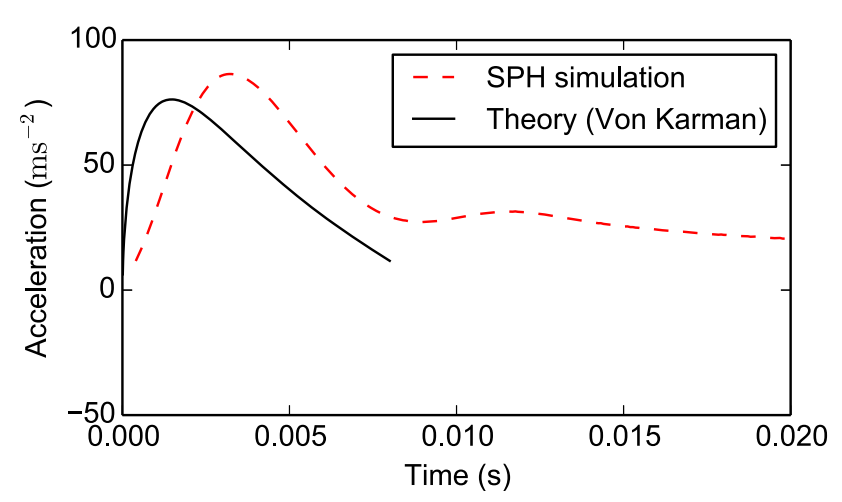

Figure 19. Vertical acceleration of sphere on water entry: simulation and theory [40].

where $V_{0}$ is the velocity with which the sphere impacts the water surface. The mass of the sphere is given by $m$ and $f(a)$ is the term that accounts for the added mass effect when a plate impacts a water surface parallel to it. The variables used in the derivation are presented in the schematic of figure 20. The radius of the sphere is given by $r$. During the impact, $y$ represents the depth of immersion of the sphere beneath the water surface and $a$ denotes the radius of the circle formed at the intersection of the sphere and the water surface. We have

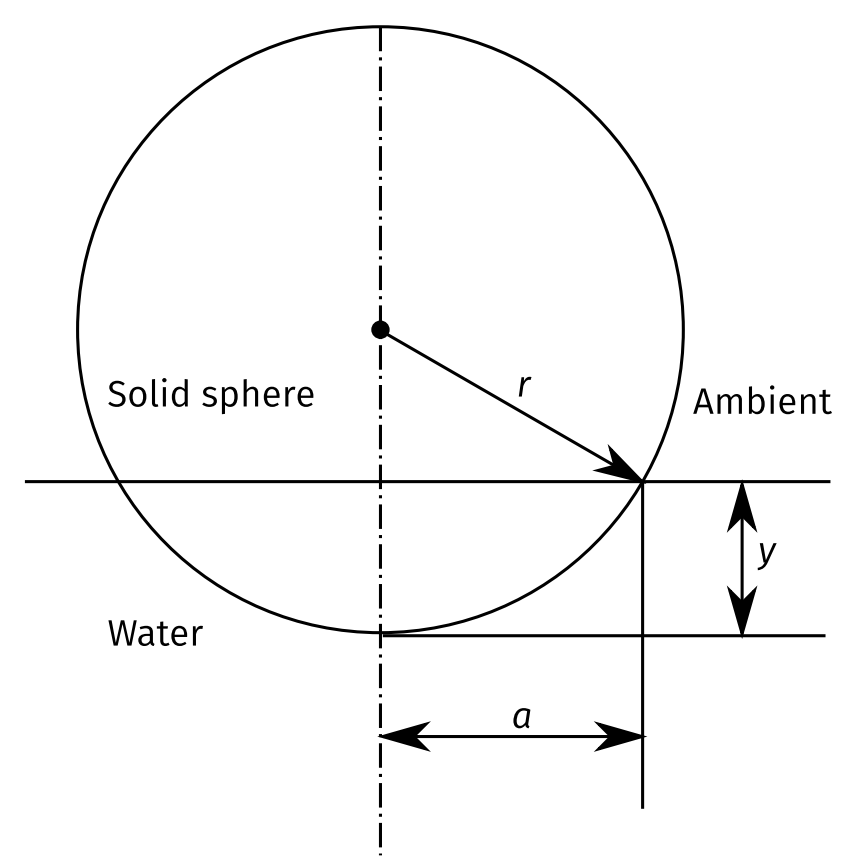

Figure 20. Schematic of water entry of sphere with the geometric notations.

$$
V=\frac{V_{0}}{1+f(a) / m} .
$$

A volume corresponding to $64 \%$ of the volume of a sphere is assumed to be 'added' by a flat plate of the same radius. The added mass is then

$$
0.64 \rho \frac{4}{3} \pi a^{3}
$$

For the impact at a free surface, the added mass can be assumed to be half of the above, accounting for one side of the plate alone. Therefore

$$
f(a)=0.32 \rho \frac{4}{3} \pi a^{3} .
$$

Now, we have

$$
V=\frac{d y}{d t}=\frac{V_{0}}{1+f(a) / m}
$$

and

$$
\frac{d^{2} y}{d t^{2}}=-\frac{V_{0}}{m}\left(1+\frac{f(a)}{m}\right)^{-2} \frac{d f(a)}{d x} \frac{d x}{d t} .
$$

From the geometry of the sphere shown earlier

$$
a^{2}=2 r y-y^{2}
$$

Using this and simplifying, we have

$$
\frac{d^{2} y}{d t^{2}}=-\frac{V_{0}}{m} \frac{1.28 \rho \pi a(r-y) \frac{d y}{d t}}{\left(1+\frac{1.28}{3 m} \pi \rho a^{3}\right)^{2}}
$$


where $a=\sqrt{\left(2 r y-y^{2}\right),} m$ is the mass and $V_{0}$ is the velocity of the sphere at impact. The differential equation is solved numerically using second-order explicit RungeKutta method, and the result is used for comparison in figure 19. Note that the initial steep rise, as predicted by theory, is due to the assumption that the sphere is made of layers of circular plates and this does not account for the 3D shape of the sphere itself. However the maximum deceleration experienced by the sphere upon impact is well predicted by the SPH method. The simulation shows a reasonable agreement with this acceleration limit.

\section{Conclusions}

We employed an improved free surface algorithm for Incompressible Smoothed Particle Hydrodynamics together with a rigid body dynamics algorithm to simulate the impact of solids into water. After validating the free surface profile and the penetration dynamics of a neutrally buoyant rigid body against experimental results available in literature, we performed a parametric study of water entry of $2 \mathrm{D}$ solids (cylinders) of different shapes, different imparted angular velocities and densities. The total kinetic energy of the falling solids was maintained the same for the different shapes and the different angular velocity cases. We presented a comparison of the impact pressure profile, vertical velocity, angular velocity, kinetic energy of the solid and the energy imparted to the liquid for these various cases. We simulated a 3D sphere at low Froude number and compared the impact pressure profile with a theoretical estimate. The free surface features of the splash were also discussed in all the cases.

\section{References}

[1] Yan H, Liu Y, Kominiarczuk J and Yue D K P 2009 Cavity dynamics in water entry at low froude numbers. J. Fluid Mech. 641: 441-461

[2] Worthington A M and Cole R S 1897 Impact with a liquid surface, studied by the aid of instantaneous photography. Philos. Trans. R. Soc. A 189: 137-148

[3] Gilbarg D and Anderson R A 1948 Influence of atmospheric pressure on the phenomena accompanying the entry of spheres into water. J. Appl. Phys. 19(2): 127-139

[4] May A and Woodhull J C 1948 Drag coefficients of steel spheres entering water vertically. J. Appl. Phys. 19(12): $1109-1121$

[5] May A 1951 Effect of surface condition of a sphere on its water-entry cavity. J. Appl. Phys. 22(10): 1219-1222

[6] May A 1952 Vertical entry of missiles into water. J. Appl. Phys. 23(12): 1362-1372

[7] Richardson E G 1948 The impact of a solid on a liquid surface. Proc. Phys. Soc. 61(4): 352
[8] Glasheen J W and McMahon T A 1996 A hydrodynamic model of locomotion in the basilisk lizard. Nature 380(6572): 340-341

[9] Holland K T, Green A W, Abelev A and Valent P J 2004 Parameterization of the in-water motions of falling cylinders using high-speed video. Exp. Fluids 37(5): 690-700

[10] Chu P C, Fan C, Evans A D and Gilles A 2004 Triple coordinate transforms for prediction of falling cylinder through the water column. J. Appl. Mech. 71(2): 292-298

[11] Truscott T T, Epps B P and Belden J 2014 Water entry of projectiles. Annu. Rev. Fluid Mech. 46: 355-378

[12] Techet A H and Truscott T T 2011 Water entry of spinning hydrophobic and hydrophilic spheres. J. Fluid. Struct. 27(5): 716-726

[13] Truscott T T and Techet A H 2006 Cavity formation in the wake of a spinning sphere impacting the free surface. Phys. Fluids 18(9): 91113-91113

[14] Truscott T T and Techet A H 2009 A spin on cavity formation during water entry of hydrophobic and hydrophilic spheres. Phys. Fluids 21(12): 121703

[15] Truscott T, Belden J and Hurd R 2014 Water-skipping stones and spheres. Phys. Today 67(12): 70-71

[16] Birkhoff G et al 2012 Jets, wakes, and cavities. Elsevier, Amsterdam

[17] Ahmadzadeh M, Saranjam B, Hoseini Fard A and Binesh A R 2014 Numerical simulation of sphere water entry problem using Eulerian-Lagrangian method. Appl. Math. Model. 38(5-6): 1673-1684

[18] Gingold R A and Monaghan J J 1977 Smoothed particle hydrodynamics - theory and application to non-spherical stars. Mon. Not. R. Astron. Soc. 181: 375-389

[19] Monaghan J 1985 Particle methods for hydrodynamics. Comput. Phys. Rep. 3(2): 71-124

[20] Monaghan J J 2005 Smoothed particle hydrodynamics. Rep. Prog. Phys. 68(8): 1703

[21] Cummins S J and Rudman M 1999 An SPH projection method. J. Comput. Phys. 152(2): 584-607

[22] Nair P and Tomar G 2015 Volume conservation issues in incompressible smoothed particle hydrodynamics. J. Comput. Phys. 297: 689-699

[23] Nair P and Tomar G 2014 An improved free surface modeling for incompressible SPH. Comput. Fluids 102: 304-314

[24] Greenhow M and Lin W-M 1983 Nonlinear-free surface effects: experiments and theory. Technical Report, DTIC Document

[25] Truscott T T and Techet A H 2009 Water entry of spinning spheres. J. Fluid. Mech. 625: 135-165

[26] Aristoff J M, Truscott T T, Techet A H, and Bush J W M 2010 The water entry of decelerating spheres. Phys. Fluids 22(3): 032102

[27] Antuono M, Colagrossi A, Le Touzé D and Monaghan J J 2013 Conservation of circulation in SPH for 2D free-surface flows. Int. J. Numer. Methods Fluids 72(5): 583-606

[28] Dehnen W and Aly H 2012 Improving convergence in smoothed particle hydrodynamics simulations without pairing instability. Mon. Not. R. Astron. Soc. 425(2): 1068-1082

[29] Colagrossi A, Souto-Iglesias A, Antuono M and Marrone S 2013 Smoothed-particle-hydrodynamics modeling of dissipation mechanisms in gravity waves. Phys. Rev. E 87(2): 023302

[30] Monaghan J J 1994 Simulating free surface flows with SPH. J. Comput. Phys. 110(2): 399-406 
[31] Cole R H 1948 Underwater explosions. Princeton, NJ: Princeton University Press

[32] Lee E S, Moulinec C, Xu R, Violeau D, Laurence D and Stansby P 2008 Comparisons of weakly compressible and truly incompressible algorithms for the SPH mesh free particle method. J. Comput. Phys. 227(18): 8417-8436

[33] Nishida A 2010 Experience in developing an open source scalable software infrastructure in Japan. In: Proceedings of Computational Science and Its Applications-ICCSA 2010, p. 448-462. Springer, Berlin

[34] Shao S and Lo E Y M 2003 Incompressible SPH method for simulating Newtonian and non-Newtonian flows with a free surface. Adv. Water Resour. 26: 787-800

[35] Khayyer A, Gotoh H and Shao S D 2008 Corrected incompressible SPH method for accurate water-surface tracking in breaking waves. Coast. Eng. 55: 236-250
[36] Rafiee A and Thiagarajan K P 2009 An SPH projection method for simulating fluid-hypoelastic structure interaction. Comput. Method. Appl. Mech. Eng. 198(33-36): 2785-2795

[37] Gotoh H and Sakai T 2006 Key issues in the particle method for computation of wave breaking. Coast. Eng. 53(2): 171-179

[38] Koshizuka S, Nobe A and Oka Y 1998 Numerical analysis of breaking waves using the moving particle semi-implicit method. Int. J. Numer. Methods Fluids, 26: 751-769

[39] Bøckmann A, Shipilova O and Skeie G 2012 Incompressible SPH for free surface flows. Comput. Fluids 67: 138-151

[40] von Kármán T 1929 The impact on seaplane floats during landing. National Advisory Committee on Aeronautics

[41] Hoover W R and Dawson V C D 1966 Hydrodynamic pressure measurements of the vertical water entry of a sphere. Technical Report, DTIC Document 